treatment sequencing and outcomes from six centres across Europe and South Korea.

Methodology This retrospective cohort study used longitudinal data collected from electronic medical records from 6 European and South Korean treatment centres. A standard protocol \& common data model was developed to capture consistent data for patients diagnosed between January 2012 and December 2018, with a minimum of 12 months follow-up. Full treatment data was collected and categorized in programmes of care based on exemplar patient narratives. An extensive data harmonization process was implemented to ensure different country and site medical records were interpreted in a common manner. Overall survival (OS) and time to next treatment (TTNT) were estimated using Kaplan Meier methodology and outcomes stratified by categories of interest. Each site analysed their own EMR data and shared aggregated results for comparison.

Results Table 1 demonstrates patient characteristics from six sites. In total the overall study cohort includes 2889 patients. Median age for each centre ranged from 53 to 67 years. The majority of patients were FIGO stage III (range 31\% to 66\%) and high-grade serous morphology (52\% to 69.9\%) Additional data on treatment pathways and outcomes for each centre will be presented.

Conclusion Preliminary analysis from this network suggests a consistent profile of adults treated for EOC across most contributing treatment centres in Europe, but with some substantial differences compared to patients treated at centres in South Korea and Romania. The establishment of a common data model between sites across five different countries allows for detailed exploration of the factors influencing differences in patient management and treatment outcomes in ovarian cancer patients.

Disclosures Sue Cheeseman: I receive consultancy fees from IQVIA

Bethany Levick: I am an employee of IQVIA

Eunji Nam: I have no conflict of interest to disclose

Dongkyu Kim: I have no conflict of interest to disclose

Roman Rouzier: I have no conflict of interest to disclose

Claire Bonneau: I have no conflict of interest to disclose

Paul Kubelac: served on a speaker's bureau for Roche, Bristol-Myers Squibb, AstraZeneca, Novartis.

Patriciu Achimas-Cadariu: I have no conflict of interest to disclose

Jean-Marc Classe: I have no conflict of interest to disclose

François Bocquet: I have no conflict of interest to disclose

Sven Becker: I have no conflict of interest to disclose

Mariana Guergova-Kuras: I am an employee of IQVIA

Geoff Hall: I receive grant support and consultancy fees from IQVIA

\section{MAINTENANCE OLAPARIB IN PATIENTS WITH NEWLY DIAGNOSED ADVANCED OVARIAN CANCER AND A BRCA MUTATION: SUBGROUP ANALYSIS BY RISK IN THE PHASE III SOLO1 STUDY}

${ }^{1}$ Nicoletta Colombo, ${ }^{2}$ William Bradley, ${ }^{3}$ Kathleen Moore, ${ }^{4}$ Antonio González-Martín ${ }^{5}$ Giovanni Scambia, ${ }^{6}$ Ana Oaknin, ${ }^{7}$ Michael Friedlander, ${ }^{8}$ Elizabeth Lowe, ${ }^{8}$ Phil Rowe, ${ }^{9}$ Paul Disilvestro. ${ }^{1}$ University of Milan-Bicocca and Istituto Europeo DI Oncologia; ${ }^{2}$ Froedtert and the Medical College of Wisconsin; ${ }^{3}$ Stephenson Oklahoma Cancer Center; ${ }^{4}$ Clínica Universidad de Navarra; ${ }^{5}$ Università Cattolica del Sacro Cuore-Fondazione Policlinico A. Gemelli, Irccs; ${ }^{6}$ Vall D'hebron University Hospital, Vall D'hebron Institute of Oncology (Vhio); ${ }^{7}$ University of New South Wales Clinical School, Prince of Wales Hospital; ${ }^{8}$ Astrazeneca; ${ }^{9}$ Women and Infants Hospital

10.1136/ijgc-2020-ESG0.130
Introduction/Background In the Phase III SOLO1 study (NCT01844986) of patients with newly diagnosed advanced ovarian cancer and a BRCA mutation (BRCAm), maintenance olaparib significantly improved investigator-assessed progression-free survival (PFS) versus placebo (hazard ratio [HR] 0.30 , 95\% CI 0.23-0.41; P $<0.001 ;$ Moore et al. $\mathrm{N}$ Engl J Med 2018). We present an exploratory analysis from higherrisk and lower-risk subgroups.

Methodology Patients received olaparib $300 \mathrm{mg}$ twice daily or placebo for up to 2 years or until disease progression. In this exploratory analysis (primary data cut-off: 17 May 2018), patients in the higher-risk group had stage IV disease, stage III disease with residual disease following primary debulking surgery, inoperable stage III disease, or had stage III disease and underwent interval surgery. Patients in the lower-risk group had stage III disease without residual disease following primary debulking surgery. PFS was assessed by investigators and blinded independent central review (BICR). Response was assessed using modified RECIST v1.1.

Results Of 391 patients, 56\% were higher risk and 44\% were lower risk. After a median follow-up overall of $\sim 41$ months, the risk of disease progression/death per investigator was significantly reduced with olaparib versus placebo in the higherrisk group (HR 0.34, 95\% CI $0.24-0.48 ; 66 \%$ reduction) and the lower-risk group (HR 0.33, 95\% CI 0.20-0.52; 67\% reduction) (figure 1). Investigator-assessed median PFS was 39.0 versus 11.1 months for olaparib versus placebo, respectively in the higher-risk group, and not reached (NR) versus 21.9 months in the lower-risk group (figure 1). Results were similar per BICR (table 1).

Conclusion In this exploratory analysis of data from the SOLO1 study, maintenance olaparib provided a substantial PFS benefit over placebo in patients with both higher-risk and lower-risk newly diagnosed advanced ovarian cancer and a BRCAm, with an investigator-assessed median PFS of 39 months and NR with olaparib treatment in higher-risk and lower-risk groups respectively after a median follow-up of $\sim 41$ months. SOLO1 is the only trial of maintenance monotherapy with a PARP inhibitor to have demonstrated a consistently high reduction in the risk of progression/death in both higher-risk and lower-risk patients with newly diagnosed advanced ovarian cancer.

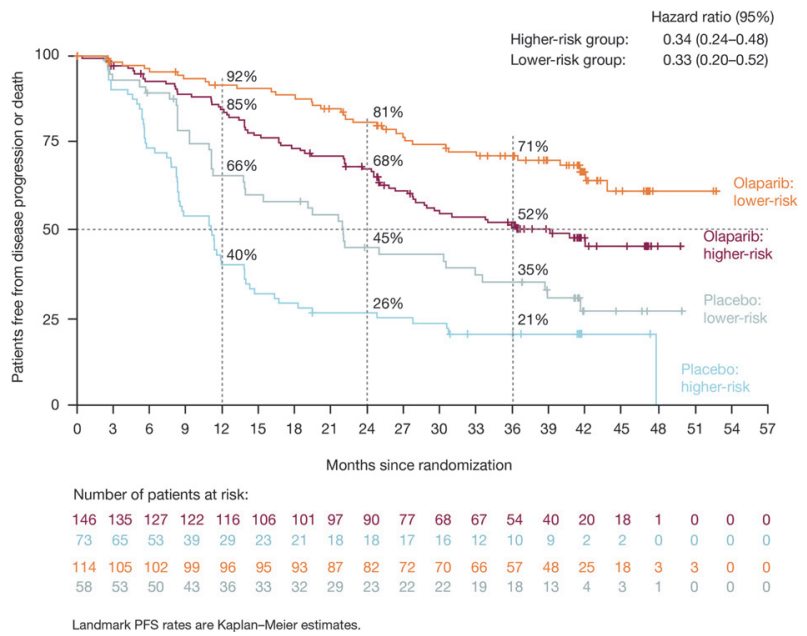

Abstract 392 Figure 1 Investigator-assessed PFS in higher-risk and lower-risk SOLO1 subgroups 
Abstract 392 Table 1 PFS in higher-risk and lower-risk SOLO1 subgroups by investigator and BICR assessment

\begin{tabular}{|c|c|c|c|}
\hline & \multicolumn{2}{|c|}{ Median PFS, months } & \multirow{2}{*}{$\begin{array}{c}\text { HR }(95 \% \mathrm{Cl}) \\
\text { Olaparib vs Placebo }\end{array}$} \\
\hline & Olaparib & Placebo & \\
\hline \multicolumn{4}{|l|}{ Investigator } \\
\hline Higher risk & 39.0 & 11.1 & $0.34(0.24-0.48)$ \\
\hline Lower risk & NR & 21.9 & $0.33(0.20-0.52)$ \\
\hline \multicolumn{4}{|l|}{ BICR } \\
\hline Higher risk & NR & 11.3 & $0.32(0.22-0.49)$ \\
\hline Lower risk & NR & 19.2 & $0.29(0.17-0.50)$ \\
\hline
\end{tabular}

Disclosures Nicoletta Colombo: Consulting fees or advisory role from Roche/Genentech, PharmaMar, AstraZeneca, Clovis Oncology, Pfizer, MSD Oncology, Takeda, Tesaro, BioCad and GlaxoSmithKline; honoraria from Roche/Genentech, AstraZeneca, Tesaro and PharmaMar. William Bradley: Nothing to disclose. Kathleen Moore: Advisory board fees from Advaxis, Aravive, AstraZeneca, Clovis, Eisai, Genentech/ Roche, Immunogen, Janssen, Merck, Oncomed, Pfizer, Samumed, Tesaro, VBL Therapeutics. Antonio González-Martín: Speaker role/consulting fees from AstraZeneca, PharmaMar, Roche, Tesaro; consulting fees from Clovis Oncology, Genmab, Inmunogen, MSD, Pfizer. Giovanni Scambia: Nothing to disclose. Ana Oaknin: Consulting fees from AstraZeneca, Clovis Oncology, Genmab, Immunogen, PharmaMar, Roche, Tesaro. Michael Friedlander: Honoraria and speaker role/advisory boards from AstraZeneca; advisory board fees from Lilly, MSD, Takeda, Novartis; non-remunerated consulting for AbbVie; research funding from Beigene, AstraZeneca, Novartis. Elizabeth S. Lowe: Shareholder and employee of AstraZeneca. Philip Rowe: Shareholder and employee of AstraZeneca. Paul DiSilvestro: Consulting fees from AstraZeneca, Tesaro.

\section{REAL-LIFE RESPONSE TO STANDARD OF CARE IN ADVANCED OVARIAN CANCER - AN OBSERVATIONAL MULTI-NATIONAL COHORT STUDY (RESPONSE)}

${ }^{1}$ Christian Marth, ${ }^{2}$ Heini Lassus, ${ }^{3}$ Kristina Lindemann, ${ }^{4}$ Els Van Nieuwenhuyen, ${ }^{5}$ Anne Weng Ekmann-Gade, ${ }^{6}$ Jesper Hansen, ${ }^{7}$ Klaus Kaae Andersen, ${ }^{8}$ Jacob Korach. ${ }^{1}$ Medical University Innsbruck; Department of Obstetrics and Gynecology; ${ }^{2}$ Helsinki University Hospital and University of Helsinki; Department of Gynecology and Obstetrics; ${ }^{3}$ Oslo Universitetssykehus, Kreftklinikken; Avdeling for Gynekologisk Kreft; ${ }^{4}$ University Hospital Leuven; Department of Obstetrics and Gynecology; ${ }^{5}$ Juliane Marie Centre, Rigshospitalet; Department of Gynaecology; ${ }^{6}$ Astrazeneca; Medical Affairs; ${ }^{7}$ Astrazeneca; Medical Evidence; ${ }^{8}$ Sheba Medical Center

\subsection{6/ijgc-2020-ESGO.131}

Introduction/Background Recent randomized clinical trials have demonstrated convincing effects of integrating PARP inhibitors (PARPi) and combination of PARPi + bevacizumab (antiVEGF) into first line (1L) treatment of selected groups of advanced stage ovarian cancer (OC) patients. However, it remains unclear to which extent eligibility of PARPi treatment translates into a real-world setting, where the impact of patient heterogeneity and differences in national clinical practices may influence the potential for PARPi treatment. The aim of this study is to describe treatment strategies and outcomes of advanced OC; and to estimate the proportion of patients potentially eligible for 1L PARPi maintenance therapy and for concomitant anti-VEGF treatment practice using observational data in a multi-national setting (RESPONSE).

Methodology This international, multi-centre, observational study, includes real-world data on diagnostic work-up, standard of care, clinical outcomes and treatment for around 1000 patients with advanced OC $(\geq 120$ patients/country). Last index date is 1st April 2018, ensuring at least 20 months of follow-up. Potential PARPi eligibility is defined as having no macroscopic residual disease $(<1 \mathrm{~cm})$ following upfront surgery and/or having a clinical complete response/partial

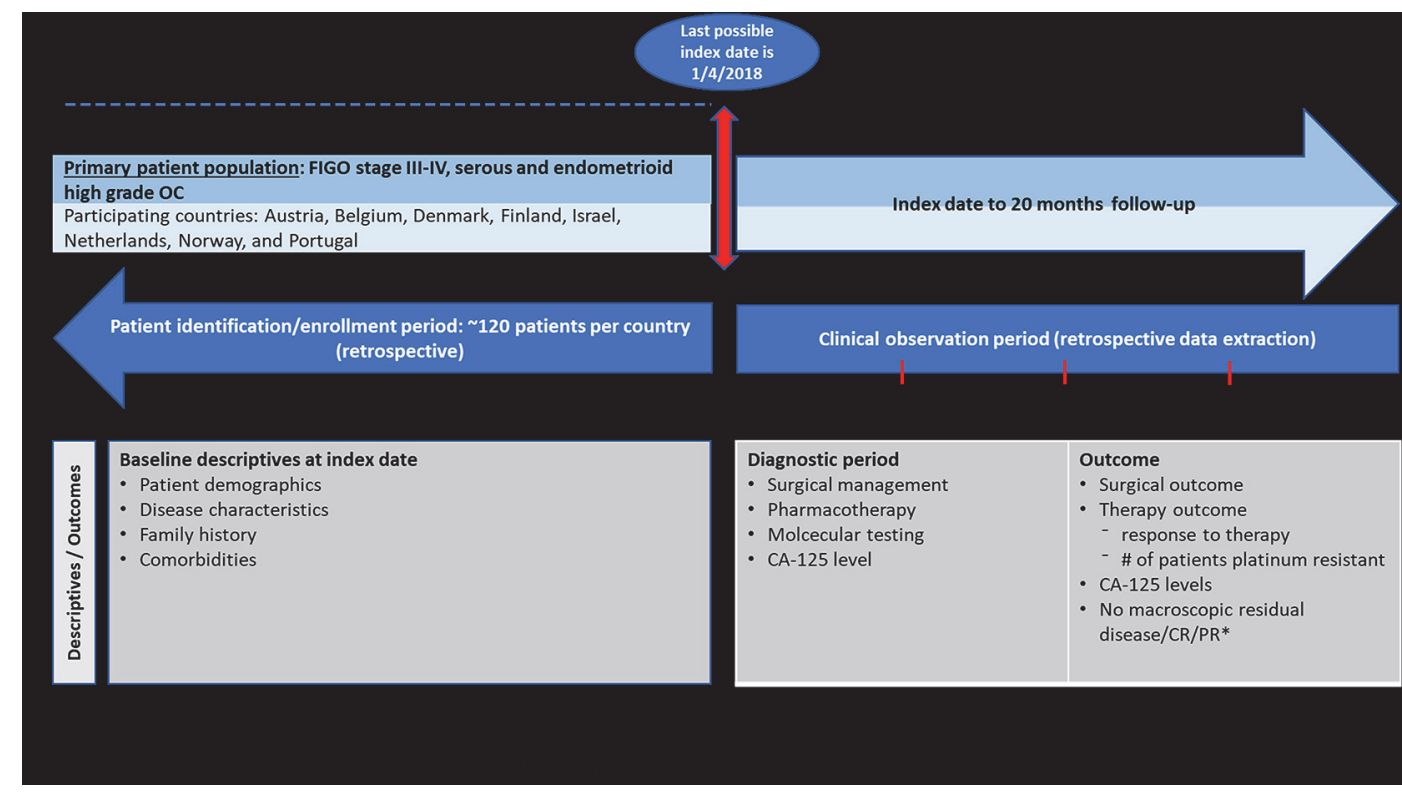

Abstract 405 Figure 1 